\title{
GÉNERO, CULTURA Y DESEMPEÑO EN MATEMÁTICAS
}

Alejandra Mizala Salces 


\section{ALEJANDRA MIZALA SALCES}

Economista de la Universidad de Chile y Ph.D en Economía de la Universidad de California, Berkeley. Profesora Titular de la Facultad de Ciencias Físicas y Matemáticas. Actualmente es Directora del Instituto de Estudios Avanzados en Educación y del Centro de Investigación Avanzada en Educación (CIAE), así como miembro del Centro Economía Aplicada de Ingeniería Industrial de la Universidad de Chile. 


\section{GÉNERO, CULTURA Y DESEMPEÑO EN MATEMÁTICAS ${ }^{1}$}

\section{INTRODUCCIÓN}

En muchos países, las mujeres participan menos que los hombres en las ciencias exactas y las tecnologías. En el 2014, en los países de la OECD sólo uno de cada cuatro estudiantes en primer año de ingeniería era mujer (24\%) y sólo el 37\% de los estudiantes de primer año en ciencias, matemáticas e informática era mujer; aún más, sólo el 19\% de los matriculados en primer año de informática era del género femenino. Pero incluso dentro de las ciencias existe segmentación por género, de acuerdo a los datos recogidos al aplicar la prueba PISA, la que incluye a 50 países: en todos ellos, las niñas orientadas a las ciencias mostraron preferencias por biología, agricultura y carreras de la salud, mientras que los niños prefirieron carreras en informática, ingeniería y matemáticas. Estas diferencias se observan aún entre los estudiantes con mejores resultados académicos (Sikora y Pokropek, 2012a).

En Chile, la matrícula de mujeres en las universidades es mayor que la de los hombres, fenómeno que ocurre desde hace varios años y que se verifica también en el resto de las instituciones de educación superior. Las mujeres también superan a los hombres en las tasas de titulación, tendencia que es creciente. En los doctorados nacionales, por su parte, un 44\% de los matriculados en el periodo 2007-2015 fueron mujeres y si bien el porcentaje de mujeres en doctorados de ingeniería y ciencias es más alto que el de mujeres matriculadas en carreras de pregrado en esas mismas áreas, esta proporción es significativamente menor que la de los hombres.

Las desigualdades de género que podemos detectar en el sistema de educación superior están vinculadas y son producto de una serie de desigualdades a lo largo del ciclo de vida de hombres y mujeres. A pesar de que no se observan diferencias en los resultados académicos en los primeros años de educación -por ejemplo, niñas y niños tienen resultados similares en la prueba SIMCE de matemáticas en $4^{\circ}$ año de enseñanza básica-, en los cursos finales de la educación básica ya se aprecian brechas, las que tienden a agudizarse con el tiempo y perjudican a las mujeres.

1. Se agradece el financiamiento otorgado por el Proyecto Basal FB0003 del Programa de Investigación Asociativa de CONICYT. 
Las brechas de género en matemáticas están siendo ampliamente estudiadas porque tienen impacto en la elección de carreras y más tarde en los salarios que hombres y mujeres obtienen en el mercado laboral. Investigaciones recientes muestran que gran parte de las diferencias observadas pueden atribuirse a diferencias socioculturales y a situaciones de discriminación de las mujeres en la sociedad. En particular, hay estudios internacionales que analizan a estudiantes con talento en resolución de problemas matemáticos y muestran que el desarrollo de estos talentos en mujeres depende de las características de los sistemas educativos y de la equidad de género en la sociedad, y no de diferencias biológicas entre hombres y mujeres. En esto juegan un rol relevante los hogares y el sistema educacional.

En el caso de los hogares, se ha observado que las madres juegan un papel importante en la trasmisión de expectativas de rol y que los estereotipos de género hacia las matemáticas inciden en la autopercepción que tienen las niñas sobre su propia habilidad. También se ha observado que los estímulos que los niños y las niñas reciben, por ejemplo, a través de los juguetes o los juegos que realizamos con ellos, pueden ser de distinta naturaleza y así afectar el desarrollo de determinadas habilidades.

En cuanto a los establecimientos educacionales, existe evidencia de que los estereotipos de género hacia las matemáticas ya están presentes en los primeros años de educación y que pueden afectar el desempeño de las niñas a través de una mayor ansiedad en matemáticas ${ }^{2}$. A su vez, los profesores tienen expectativas diferenciadas en relación al posible rendimiento de sus alumnos hombres y mujeres en matemáticas, lo que puede afectar los procesos de acumulación de habilidades en matemáticas, generando una profecía autocumplida.

En este artículo se presenta evidencia disponible para Chile respecto de este tema y se resumen resultados de investigaciones realizadas con otros colegas y estudiantes que buscan comprender la fuente de las brechas de género en educación.

\section{REVISIÓN DE LA LITERATURA}

El estudio de las brechas de género en habilidad matemática en distintos contextos muestra que muchas de las diferencias observadas pueden atribuirse a diferencias socioculturales y a las situaciones de discriminación de las mujeres en la sociedad. Else-Quest et al. (2010) muestran, en un meta análisis basado en estudios de las

2. Ansiedad en matemáticas: Sensación de tensión y ansiedad que interfiere con la manipulación de problemas matemáticos en un espectro amplio de situaciones cotidianas y académicas (Richardson y Suinn, 1972). 
pruebas internacionales TIMSS y PISA, que la variabilidad entre países de las diferencias de género reportadas en matemáticas está asociada a características culturales de los países: aquellos con mayor equidad de género tienen las menores brechas en matemáticas. Fryer y Levitt (2010) y Guiso et al. (2008) -quienes realizan un análisis entre países usando resultados de la prueba PISA- llegan a una conclusión similar: la brecha de género en el desempeño en matemáticas se correlaciona con oportunidades económicas y sociales de las mujeres en los países, por ejemplo, con la tasa de participación laboral femenina, el porcentaje de mujeres en altos cargos de empresas, de gobierno y la participación parlamentaria femenina.

Hyde y Mertz (2009) muestran que en muchos sistemas educativos las mujeres no sólo alcanzan a los hombres en cuanto a resultados promedio en matemáticas sino que también se encuentran mujeres en los niveles más altos de habilidad en matemáticas. De hecho, en algunos de los países y economías con mejor desempeño en la prueba PISA, las niñas obtienen el mismo puntaje o puntajes más altos que sus compañeros de clase en matemáticas, como es el caso de Hong Kong, Shanghai, Singapur y Taipei-China. Esto sugiere que la brecha de género en matemáticas no está determinada por diferencias innatas en la capacidad de hombres y mujeres.

Algo similar concluyen Andreescu et al. (2007) en su análisis internacional de estudiantes con talento en resolución de problemas matemáticos: existen mujeres talentosas en matemáticas y la aparición de estos talentos depende más de las características de los sistemas educativos y de la equidad de género en la sociedad que de diferencias biológicas entre hombres y mujeres. Por otro lado, Lindberg et al. (2010), en un meta-análisis de 242 investigaciones publicadas entre 1990 y 2007, concluyen que no se observan diferencias de género ni en los resultados promedio ni en la variabilidad de estos.

En definitiva, la evidencia más reciente muestra que aunque existan diferencias biológicas entre hombres y mujeres, ambos sexos comparten la misma base biológica para la comprensión y dominio del conocimiento matemático, aún al más alto nivel. El desarrollo de estos talentos en mujeres depende de las características de los sistemas educativos y de la equidad de género en la sociedad.

\section{Estereotipos de género en hogares y establecimientos educacionales}

Existe evidencia sobre el impacto de los estereotipos de género de padres y profesores, así como de sus expectativas, sobre las actitudes de niños, niñas y jóvenes hacia las matemáticas (Gunderson et al., 2012).

En el caso de los hogares, se ha observado que las madres juegan un rol importante en la trasmisión de expectativas de rol (González de San Román y de la Rica Goiricelaya, 2012) y que los estereotipos de género hacia las matemáticas de las 
madres inciden en la autopercepción que tienen las niñas sobre su propia habilidad (Tomasetto et al., 2015). Por otro lado, a pesar de que existe evidencia contradictoria sobre el tiempo que dedican los padres a hijos e hijas (por separado) en términos de inversión en habilidades cognitivas (Baker y Milligan, 2013; Hong et al., 2010), se ha observado que los niños reciben estímulos que pueden ser de distinta naturaleza, lo que podría afectar los resultados (Gunderson et al., 2013).

En cuanto a la escuela, existe evidencia de que los estereotipos de género hacia las matemáticas ya están presentes en los primeros años de educación primaria (Cvencek et al., 2011) y que estos pueden afectar el desempeño de las mujeres a través de una mayor ansiedad en matemáticas (Maloney y Beilock, 2012). Además, la autoconfianza en matemáticas es menor en las niñas (Ahmed et al., 2012; Bharadwaj et al., 2015), lo que puede afectar los procesos de acumulación de habilidades en matemáticas: menores niveles de autoconfianza pueden generar creencias erradas sobre las propias habilidades en grupos discriminados e interrumpir los procesos de acumulación de capital humano (Filippin y Paccagnella, 2012), generando una profecía auto cumplida.

\section{Estereotipos de género y resultados en pruebas de matemáticas}

El impacto de los factores anteriores es significativo, sobre todo si se considera que las mujeres ingresan al sistema escolar con mejores habilidades cognitivas que los hombres (Buchmann et al., 2008), tienen un desarrollo cognitivo que precede a estos en la pubertad (Zahn-Waxler et al., 2008) y, en general, exhiben mayores niveles de asistencia escolar, mejor conducta y compromiso con las tareas escolares, lo que es valorado por el sistema escolar y se traduce en mejores notas (Di Prete y Jennings, 2009). En los hechos, las mujeres muestran resultados inferiores en pruebas en donde todo indica que deberían sobrepasar a los hombres.

Se ha observado que los resultados de las pruebas pueden subestimar las habilidades cognitivas reales de poblaciones negativamente estereotipadas (Walton y Spencer, 2009), como es el caso de las mujeres en el dominio de las matemáticas. La amenaza de estereotipo se define como una situación en donde el miembro de un grupo social discriminado está en riesgo de confirmar, como una característica propia real, un estereotipo negativo sobre el grupo al que pertenece (Steele y Aronson, 1995). La amenaza de estereotipo, al afectar el resultado en pruebas cognitivas, entrega una medida errónea de las verdaderas habilidades de grupos discriminados.

Nguyen y Ryan (2008) realizan un meta-análisis de 116 estudios experimentales y encuentran efectos significativos de la amenaza de estereotipo en el desempeño en pruebas. Los estudios sobre amenaza de estereotipo han indagado en los factores que 
están detrás de la relación entre amenaza y bajos resultados. Primero, la relevancia del estereotipo es relativa a la situación de evaluación ${ }^{3}$. En segundo lugar está la identificación de los individuos con el dominio evaluado. Por ejemplo, la amenaza de estereotipo debería afectar a las mujeres que se identifican con las matemáticas, no a aquellas que no tienen interés en este dominio. Tercero, a mayor nivel de dificultad de la prueba, mayor amenaza de estereotipo. En cuarto lugar está el tipo de estereotipo, el que varía entre distintos grupos negativamente estereotipados, por ejemplo, según género, nivel socioeconómico, raza, religión, migrantes y todas sus interacciones.

Por otro lado, también se han observado efectos recíprocos entre el bienestar subjetivo de los estudiantes y el logro académico (Steinmayr et al., 2016). La preocupación de los estudiantes ante las pruebas tiene un impacto negativo tanto en el logro académico como en su bienestar. Existe evidencia de que percepciones negativas respecto del propio desempeño, aun cuando sean erradas, afectan el desempeño futuro, así como la motivación de los estudiantes y su bienestar (Kim et al., 2010).

Lo que resulta interesante de la hipótesis de amenaza de estereotipo es que explicaría las brechas de género en matemáticas como un artefacto, es decir, un error en la medición producto de la interacción entre el instrumento de medición (prueba) y la situación de evaluación.

\section{Aversión al riesgo y actitudes hacia la competencia}

Una hipótesis complementaria que explica las brechas de género en matemáticas se basa en las diferencias de género observadas en la aversión al riesgo y las actitudes hacia la competencia. Existe evidencia robusta respecto de que la aversión al riesgo es mayor en las mujeres (Bertrand, 2011; Croson y Gneezy, 2009), lo que se relaciona con una mayor autoconfianza de los hombres en diferentes tareas. Estas diferencias de género en la aversión al riesgo pueden afectar los resultados de hombres y mujeres en ambientes competitivos y explicar las diferencias en pruebas de matemáticas que no se relacionan con una diferencia innata en las habilidades de hombres y mujeres (Niederle y Vesterlund, 2010).

No obstante, las diferencias de género relativas al desempeño en ambientes competitivos parecen tener una relación importante con la cultura, específicamente

3. Por ejemplo, a pesar de que existe evidencia de lo robusto de las diferencias de género en la capacidad de cognición espacial, Hyde (2014) muestra que esta evidencia proviene de tests que hacen que los participantes resuelvan problemas contra el tiempo. Cuando se elimina la presión del tiempo, hombres y mujeres exhiben resultados similares. 
con el rol de la mujer en la sociedad (Gneezy et al., 2009) ${ }^{4}$. La evidencia muestra una importante variabilidad de las brechas de género en actitudes hacia la competencia dependiendo del tipo de tarea (Dreber et al., 2014), nivel de desempeño de los participantes (Garratt et al., 2013) y el diseño institucional asociado a las mediciones (Niederle y Yestrumskas, 2008). Se ha sugerido que las diferencias en actitudes hacia la competencia podrían ser observables desde los tres años de edad (Sutter y Rützler, 2010).

Mientras que algunos estudios no encuentran diferencias de género en tareas competitivas (Dreber et al., 2011), otros sí encuentran algunas que perjudican a las mujeres en ámbitos competitivos (Datta Gupta et al., 2005; De Paola et al., 2015; Niederle y Vesterlund, 2007). Gneezy et al. (2003) presentan evidencia experimental que respalda que las mujeres pueden ser menos efectivas que los hombres en entornos competitivos mixtos, aunque pueden desempeñarse de manera similar en entornos no competitivos y mejor en entornos de un solo sexo. Al contrario, evidencia experimental ha mostrado que al participar en un torneo competitivo, los hombres aumentan su desempeño, ampliándose la brecha de género. Gneezy y Rustichini (2004) obtienen resultados similares: las evaluaciones competitivas mejoran el desempeño de los hombres mientras que el desempeño de las mujeres se mantiene estable.

La evidencia sobre género y competencia es robusta en indicar que en ambientes estereotipadamente masculinos se observa una diferencia de género en la propensión a competir entre hombres y mujeres con niveles de habilidad similar (Niederle y Vesterlund, 2011) $)^{5}$. Algunos estudios han observado específicamente el impacto de pruebas competitivas y no competitivas en las brechas de género en resultados académicos. Örs, Palomino y Peyrache (2013) y Jurajda y Münich (2011) encuentran que estudiantes de similar habilidad, medida por un test no competitivo, presentan una brecha en contra de las mujeres en test competitivos de altas consecuencias.

4. Este trabajo muestra que frente a una opción competitiva versus una no competitiva, en una sociedad patriarcal tradicional un 50\% de los hombres prefiere competir y tener un mayor premio, mientras que sólo un $26 \%$ de las mujeres escoge esta modalidad. En una sociedad matriarcal donde las mujeres tienen una posición de dominancia sólo un 39\% de los hombres elige competir y tener un premio mayor, mientras que un 54\% de las mujeres prefiere el torneo competitivo.

5. Esto tiene importantes consecuencias para el acceso de las mujeres al ámbito de carreras de educación superior en las áreas de ciencia, tecnología, ingeniería y matemáticas (STEM por su sigla en inglés), acceso que frecuentemente está mediado por pruebas competitivas. 


\section{Brecha de género y elección de carrera}

En las últimas décadas, muchos países han reducido o cerrado la brecha de género en términos de años de educación y en la asistencia a educación post secundaria. Sin embargo, como ya se mencionó, las mujeres están subrepresentadas en algunas áreas vinculadas a la tecnología, la ingeniería y las matemáticas, y sobrerrepresentadas en áreas como ciencias sociales, humanidades y educación. Esto es lo que se denomina segregación sexual horizontal.

Este fenómeno puede explicar la segregación de género en el mercado laboral debido a que el contenido de la educación representa una parte sustancial de la brecha de género en el empleo y los ingresos (Arcidiacono, 2004). En general, los sujetos estereotípicamente masculinos crean un capital humano más valioso para el trabajo y generan mayores ingresos. Por lo tanto, es relevante entender por qué las mujeres no eligen las carreras más gratificantes en términos del mercado laboral y oportunidades salariales futuras. La literatura sobre este tema aún no ha llegado a respuestas definitivas.

Existen estudios que asocian elecciones de carrera con estereotipos de género. El argumento es que los atributos específicos de género, como la aversión al riesgo, la confianza en uno mismo y la actitud hacia la competencia, contribuyen a esbozar la identidad de género. Estos atributos se desarrollan durante la infancia y afectan las elecciones de niños y niñas a lo largo de sus ciclos de vida (Sutter y Rützler, 2010; Gneezy y Rustichini, 2004).

Como ya se mencionó, una amplia literatura documenta que las mujeres generalmente tienen más aversión al riesgo que los hombres y son más propensas a rehuir la competencia. Algunos autores sugieren que estas características están relacionadas con una brecha de género en la confianza acerca de sus propias habilidades (Booth y Nolen, 2011 y 2012; Croson and Gneezy, 2009; Gneezy et al., 2003; Niederle y Vesterlund, 2007 y 2010; Datta Gupta et al., 2005). En concreto, el estudio de Kurtz-Costes et al. (2008) sugiere que las niñas tienen una menor percepción que los niños sobre sus habilidades matemáticas. Por tanto, si los cursos avanzados de matemáticas son relativamente desafiantes, esto explica por qué las mujeres a menudo optan por no participar en estos cursos.

Alternativamente, la evidencia experimental sugiere que la brecha de género en la elección de carreras universitarias se debe principalmente a las diferencias en preferencias y gustos no pecuniarios, señalando el papel de las preferencias y las interacciones sociales para explicar cómo los individuos toman decisiones. Utilizando datos experimentales de los estudiantes de la Universidad de Northwestern, Zafar (2009) encuentra que las diferencias de género en las creencias sobre las propias habilidades no explican la brecha de género en la elección de carrera, tampoco 
lo explican las diferencias de género en las creencias sobre los salarios futuros asociados con cada carrera. Por el contrario, encuentra que la brecha de género se puede explicar por diferencias de género en los gustos y preferencias por diferentes áreas de conocimiento. Bartolj y Polanec (2012) obtienen un resultado similar. Los estereotipos de género discutidos anteriormente podrían explicar por qué niños y niñas tienen diferentes preferencias educativas. Las diferencias en las actitudes y preferencias pueden afectar la importancia relativa de los beneficios pecuniarios y no pecuniarios (Turner y Bowen, 1999), es decir, los incentivos económicos no son suficientes para que las niñas se matriculen y permanezcan en los campos de estudio tradicionales masculinos (Noe, 2010).

Favara (2012) estudia el papel de la identidad social en la determinación de los comportamientos individuales y las diferencias de género en los resultados económicos. Ella integra el concepto de identidad de género en un modelo económico de elecciones educativas para probar la hipótesis de que las preferencias de los estudiantes están formadas por nociones congruentes con la identidad de género. Los estudiantes eligen su carrera de acuerdo con los rendimientos monetarios esperados y los beneficios en términos de identidad. Si un estudiante se ajusta a las normas sociales de su grupo de referencia, identificado aquí por género, recibe una utilidad indirecta (pago no pecuniario) debido a una autoimagen más gratificante. Por el contrario, violar las prescripciones de identidad de género produce una pérdida de utilidad. Ella encuentra que los estereotipos de género afectan las opciones educativas desde la edad de 14 años y su efecto es mayor para las niñas que para los niños. También encuentra evidencia de que las preferencias de género pueden ser modificadas por el medio ambiente, es decir, los establecimientos educacionales de un solo sexo llevan a los estudiantes a una opción educativa menos estereotipada, una vez que se controla por la autoselección endógena en estos establecimientos.

En la misma línea, Humlum et al. (2012), utilizando datos daneses, estiman un modelo de elección de carrera e identidad. Caracterizan dos identidades relevantes para la elección de carrera: orientación profesional y orientación social. Para una persona orientada a la carrera, esta y el trabajo son importantes para una vida significativa. Por el contrario, las personas con orientación social asignan más importancia a la cooperación, la responsabilidad social y las cuestiones sociales en general. Encuentran que estos dos factores subyacentes varían sistemáticamente con las inversiones en el nivel de educación y en el área de conocimiento. Consideran este resultado como evidencia de que los pagos de identidad son una parte importante de la toma de decisiones. De acuerdo con sus hallazgos, las elecciones educativas de los estudiantes son consistentes con sus autoimágenes. Los estudiantes con una identidad orientada a la carrera eligen de acuerdo con el incentivo financiero que 
está asociado con su elección. Este hallazgo implica que los diseñadores de políticas y las instituciones de educación superior deben considerar seriamente los temas relacionados con la identidad para atraer a estudiantes de alta capacidad a ciertas carreras.

Finalmente, Sikora y Pokropek (2012) encuentran que las transferencias intergeneracionales de preferencias por carreras científicas varían considerablemente entre los países, pero existen ciertas regularidades. En muchos países, si el padre trabaja en ciencias aumenta el interés de los hijos por carreras científicas, independientemente de su campo. En contraste, el empleo materno inspira a hijas en menos países y la influencia tiende a limitarse a la biología, la agricultura y las carreras de la salud.

En las siguientes secciones se presentan investigaciones en Chile acerca de las expectativas de los futuros docentes, el efecto diferenciado que tienen sobre hombres y mujeres las pruebas competitivas de alto impacto y diferencias de género en las postulaciones a la universidad.

\section{EXPECTATIVAS DE LOS FUTUROS DOCENTES ${ }^{6}$}

Una investigación realizada con otros colegas en el marco de un proyecto Fondecyt se enfocó en estudiantes de Pedagogía Básica y ha permitido entender mejor cómo los estereotipos de género se reproducen de manera inconsciente en los futuros profesores, lo que puede afectar sus decisiones pedagógicas.

El estudio utilizó una metodología de casos hipotéticos aplicada a 208 estudiantes de Pedagogía Básica de 17 universidades a través de experimentos por encuestas (survey experiments) (Tournaki, 2003; Auwarter y Aruguete, 2008 a,b). La muestra incluyó a 176 mujeres y 32 hombres. El 41,5\% estaba en su primer año de estudios, el $26,1 \%$ en segundo año y el resto en otros años de la carrera de Pedagogía. A los futuros docentes se les presentaron casos hipotéticos de un/a alumno/alumna con bajo rendimiento en matemáticas y dificultades en conducta, y se les pedía completar un cuestionario acerca de este estudiante como si fuera su alumno. El género del estudiante y su estatus socioeconómico eran cambiados sistemáticamente, el caso planteado era idéntico y sólo cambiaba el nombre del estudiante (Marcelo o Marcela) y la profesión de su padre y madre (ejemplo: abogado/médico, obrero/asesora del hogar). A cada estudiante de Pedagogía Básica le tocaba aleatoriamente uno de los

6. Esta sección está basada en Mizala, Martínez y Martínez (2015). 
cuatro casos posibles: alumnos de alto y bajo nivel socioeconómico y alumnas de alto y bajo nivel socioeconómico?

Se les preguntó a los estudiantes de Pedagogía Básica sobre sus expectativas de rendimiento futuro de dichos alumnos en matemáticas y en todas las asignaturas, con aseveraciones como "En dos años más este estudiante aún tendrá problemas en matemáticas" en el primer caso, o "Este estudiante tendrá un mal desempeño en las pruebas estandarizadas" en el segundo caso.

El estudio encontró que las expectativas sobre el rendimiento futuro diferían significativamente en función del género del estudiante. Es decir, los futuros docentes consideraban que los hombres tendrían a futuro mejor desempeño en matemáticas que las mujeres. Además, los docentes encontraron que sólo en los casos de las niñas estas dificultades podrían tener consecuencias a largo plazo en el rendimiento académico general.

Para verificar este resultado se repitió la metodología experimental en una nueva muestra de docentes en formación, pero se les presentaron casos hipotéticos que mostraron alumnos/as con dificultades en clases de lenguaje. No hubo efectos significativos del género de los alumnos en las expectativas de rendimiento general. En otras palabras, la extrapolación de los futuros profesores acerca de que el bajo desempeño actual está relacionado con problemas académicos en el futuro sólo ocurre en matemáticas.

Estos resultados se refieren a estudiantes de Pedagogía Básica y no es posible generalizarlos a los docentes en ejercicio. No obstante, otros estudios para Chile que analizan la interacción entre docentes y estudiantes en clases de matemáticas encuentran que los docentes prestan menor atención (menor tiempo) a las niñas, más allá del hecho de que los niños son más activos en clases. También encuentran que los docentes formulan en mayor medida preguntas que requieren procesos cognitivos complejos y dan más retroalimentación a los niños que a las niñas. Es interesante mencionar que las mayores diferencias entre niños y niñas ocurren cuando los profesores tienen menor control de la clase (Bassi, Blumberg y Mateo Díaz, 2016; Espinoza y Taut, 2015).

Por otra parte, un estudio para Chile encuentra un efecto positivo y estadísticamente significativo para las niñas que tienen una profesora de matemáticas. El trabajo muestra que este efecto se produce debido a la importancia del modelo de roles y no es producto de un sesgo de la profesora. El estudio, que evalúa el

7. En el anexo se presenta el detalle de la descripción del caso que analizaban los estudiantes de Pedagogía Básica. No se observaron diferencias por nivel socioeconómico, sólo por género. 
impacto en la prueba SIMCE de matemáticas de $8^{\circ}$ básico, no encuentra un efecto sobre los niños (Paredes, 2014).

\section{ESTEREOTIPOS DE GÉNERO Y RESULTADOS EN PRUEBAS COMPETITIVAS $^{8}$}

Con el objeto de analizar la hipótesis de que las mujeres empeoran su desempeño en situaciones competitivas de alto impacto, se llevó a cabo una investigación en la cual se analizaron los resultados en matemáticas de una muestra de mellizos dicigóticos mixtos, comparando sus resultados en una prueba competitiva de alto impacto, como lo es la Prueba de Selección Universitaria (PSU), que determina la posibilidad de acceder a la carrera y universidad de su preferencia; con los resultados de una prueba no competitiva como los es la prueba SIMCE de $2^{\circ}$ año de enseñanza media. Esta última prueba no tiene consecuencias para los estudiantes, ya que los desempeños individuales no se conocen ni los estudiantes, ni sus padres o profesores tienen esta información, sólo son públicos los resultados agregados del establecimiento educacional. Es importante además aclarar que ambas pruebas tienen el mismo enfoque: miden los conocimientos de los estudiantes en relación al currículo escolar.

En concreto, se analizó una muestra de mellizos que participó en el proceso PSU 2013 e ingresó a la universidad en marzo del 2013. Este grupo fue seleccionado porque en este caso se tenía el resultado de los mismos estudiantes en ambas pruebas. Además, en su aplicación 2010, la prueba SIMCE incluyó un cuestionario específico que entrega información acerca de las habilidades no cognitivas de los estudiantes (autoeficacia en los aprendizajes). A la información obtenida de las encuestas que responden padres y apoderados, así como los estudiantes que rinden la prueba SIMCE, se agregaron las notas de los estudiantes (promedio general, promedio en matemáticas, promedio en lenguaje) y el promedio de asistencia a clases en los años disponibles. La información recopilada permitió construir una rica base de datos para el grupo de mellizos que participó en el proceso PSU 2013 de ingreso a la educación superior.

La muestra de mellizos representa una ventaja para esta investigación, ya que a pesar de que los mellizos dicigóticos no comparten el mismo contenido genético, esta condición permite un mejor control de variables no observables a nivel del hogar. En particular, la muestra de mellizos permite considerar un efecto fijo a nivel de hogar, comparando el hombre y la mujer de la pareja de mellizos y controlando

8. Esta sección se basa en Arias, Meneses y Mizala (2017). 
de esta forma por factores no observables que podrían afectar los resultados en los tests de matemáticas (Carlin, 2005).

Al analizar la muestra de mellizos mixtos (100 parejas, 200 individuos), sólo se aprecian diferencias estadísticamente significativas en contra de las mujeres en el puntaje de la prueba PSU de matemáticas (competitiva), pero no en la prueba SIMCE de matemáticas de $2^{\circ}$ medio (no competitiva). Estos resultados se obtienen controlando -además de efectos fijos por hogar- por otras variables que afectan el desempeño educacional como, por ejemplo, autoeficacia general, autoeficacia en matemáticas, promedio de notas y notas de matemáticas o lenguaje, inversión de los padres en la educación de sus hijos, dedicación del estudiante al trabajo escolar, asistencia a clases, resultados SIMCE del establecimiento educacional al que asistieron y resultados de la prueba SIMCE anterior rendida por los estudiantes (en la ecuación que busca explicar el puntaje obtenido en la PSU). Esto no ocurre en lenguaje. En estas pruebas, si bien las mujeres muestran peores resultados en relación a los hombres en la PSU que en la prueba SIMCE, estas diferencias no son estadísticamente significativas en ninguno de los dos test.

La brecha en una prueba competitiva de altas consecuencias, como la PSU, tiene impacto en las postulaciones de las mujeres a la universidad, como veremos en la próxima sección de este trabajo.

\section{DECISIONES DE ESTUDIOS SUPERIORES ${ }^{9}$}

Desde hace más de 10 años, el porcentaje de mujeres en la matrícula universitaria de primer año supera el 50\%, alcanzando el 54\% el año 2017 (Gráfico 1). Sin embargo, como se observa en el Gráfico 2, el porcentaje de mujeres por área es muy disímil, siendo este mucho menor en ciencias, tecnología, ingeniería y matemáticas (STEM por su sigla en inglés) que en todas las demás áreas: en STEM, menos del $30 \%$ de la matrícula de primer año son mujeres. El porcentaje de mujeres es especialmente alto en el área de la salud, con un 73\% de la matrícula de primer año en 2017 correspondiente a mujeres, y educación, donde $72 \%$ son mujeres; en tanto, en ciencias sociales y humanidades el $61 \%$ de la matrícula de primer año es femenina. En arte y arquitectura, por su parte, el porcentaje de mujeres supera el $55 \%$, mientras que en administración y comercio las mujeres tienden a ser poco menos de la mitad de las matriculadas en primer año.

9. Esta sección se basa en Bordon, Canals y Mizala (2017). 
Gráfico 1. Porcentaje de mujeres en matrícula universitaria de primer año

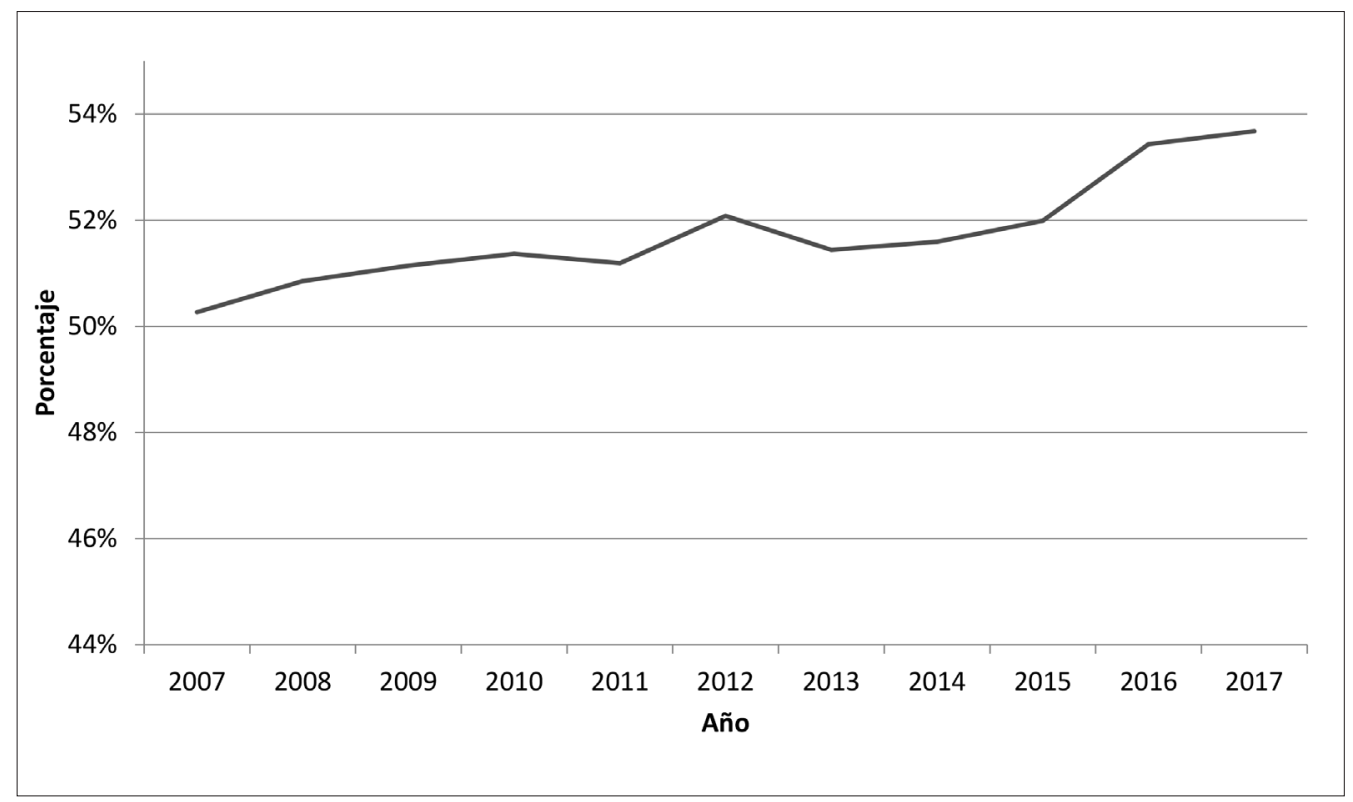

Fuente: SIES, MINEDUC

Gráfico 2. Porcentaje de mujeres en matrícula primer año de universidad según área

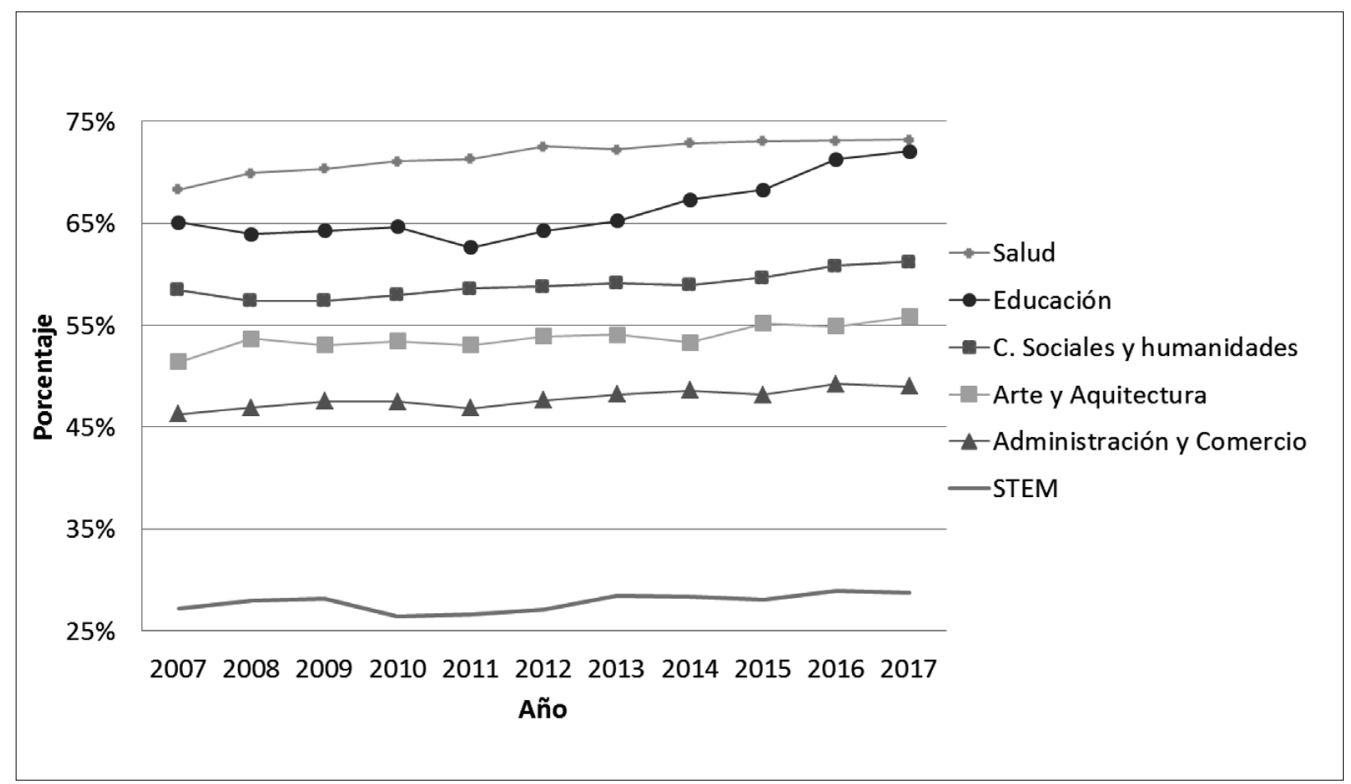

Fuente: SIES, MINEDUC 
En el área STEM no sólo hay menos mujeres sino que el aumento porcentual de la matrícula femenina en primer año entre el 2007 y 2017 fue inferior en esta área que en el resto. Mientras el porcentaje de mujeres en STEM aumentó sólo un $2 \%$ en esta década, en administración y comercio, ciencias sociales y humanidades, y arte y arquitectura aumentó entre 3\% y 4\%, en salud aumentó 5\% y en educación aumentó $7 \%$. De este modo, el crecimiento de la matrícula de las mujeres fue mayor en las áreas donde ya había más mujeres y menor donde había menos.

Para intentar entender qué está detrás de estas decisiones, estimamos un modelo guiado por las características del sistema chileno de admisión a las universidades, diferenciando las preferencias de los estudiantes del proceso de selección.

Dada la cantidad de combinaciones (carreras, universidades) a la que los estudiantes pueden postular, para estimar el modelo fue necesario trabajar con áreas de estudio en vez de carreras. Estas se agruparon en diez áreas, que corresponden a las de la clasificación CINE-UNESCO, con los siguientes cambios: ingeniería se separó de tecnología, pero las ingenierías técnicas se mantuvieron en ingeniería; medicina y odontología se separaron del resto de las carreras de la salud, ya que son más selectivas, y se agrupó ciencias sociales con humanidades. ${ }^{10}$

Por su parte, las universidades fueron organizadas en cuatro grupos, tres de los cuales corresponden a universidades del Consejo de Rectores de las Universidades Chilenas (CRUCH), las que desde hace muchos años han participado en un sistema centralizado de admisión. Estas universidades se clasifican en estos tres grupos de acuerdo a su nivel de selectividad y años de acreditación. El cuarto grupo lo componen las universidades privadas que más recientemente se han incorporado al sistema centralizado de admisión que hoy se denomina Sistema Único de Admisión (SUA). ${ }^{11}$

Estimamos un modelo logit anidado para predecir el par de elecciones académicas (área y tipo de universidad) al que postulan los estudiantes en primera preferencia ${ }^{12}$. Los datos utilizados corresponden a la cohorte que postuló a la universidad el año 2015, de la que se cuenta con datos de nivel socioeconómico de los estudiantes,

10. Las áreas consideradas son: medicina-odontología, otras carreras de la salud, ciencias básicas, ingenierías civiles, tecnología, administración y comercio, arte, ciencias sociales y humanidades, derecho y educación.

11. Universidad Adolfo Ibañez, Universidad del Desarrollo, Universidad de los Andes, Universidad Alberto Hurtado, Universidad Finis Terrae, Universidad Mayor, Universidad Andrés Bello.

12. Si bien la utilidad del estudiante y la probabilidad de ser aceptado (área, tipo de universidad) no son observables, las decisiones de los estudiantes sí lo son. En el modelo usamos el principio de las preferencias reveladas. 
el área en que trabajan o estudiaron sus padres, puntaje en la Prueba de Selección Universitaria (PSU), notas de enseñanza media (NEM) y características de los establecimientos donde estudiaron la educación media, entre otros.

Los resultados preliminares obtenidos muestran que el género afecta la elección en diferentes dimensiones.

La elección de los estudiantes tiende a reproducir la actual distribución por género de las áreas de estudio: mujeres postulan más a carreras feminizadas. Las mujeres tienen una mayor probabilidad que los hombres de postular a otras carreras de salud (14\% de mayor probabilidad), a educación y a ciencias sociales y humanidades, y menor probabilidad de postular a carreras de ingeniería (14\% menor probabilidad) y tecnología ( $8 \%$ menor probabilidad).

También se observa un fuerte efecto intergeneracional: en promedio, los postulantes tienden a reproducir el área de trabajo o estudio de los padres del mismo sexo y en menor medida el área del padre de distinto sexo. En particular, las postulantes mujeres suelen reproducir en mayor medida el área de trabajo o estudio de sus madres cuando estas se vinculan a áreas más feminizadas, tales como ciencias sociales y humanidades, arte y otras carreras de salud, esto también ocurre con la carrera de Derecho. Mientras, los padres tienen un efecto de mayor magnitud en carreras científicas (relacionadas con medicina, ciencias, ingeniería) y en educación.

Por otra parte, se encuentra que el proceso de selección es relevante: las variables NEM, PSU y la diferencia entre el puntaje individual y el puntaje de corte del año anterior son muy determinantes. De forma general, existe mayor probabilidad por parte de hombres y mujeres de postular al grupo de universidades con mejores puntajes PSU, a aquel grupo de universidades con mayor presencia en la región donde vive el estudiante y a aquel grupo de universidades donde el postulante está en mayor medida sobre el puntaje de corte del año anterior.

Sin embargo, tener mejor puntaje en la PSU de matemáticas aumenta en mayor medida la probabilidad de postular a carreras de ingeniería civil (área más selectiva vinculada a las matemáticas) en los hombres que en las mujeres. Asimismo, tener mejor puntaje en la PSU de lenguaje aumenta en mayor medida la probabilidad de postular a Derecho (carrera más selectiva vinculada al lenguaje) en los hombres que en las mujeres. De este modo, aun cuando en ambos sexos los mejores puntajes PSU en matemáticas/lenguaje se asocian a mayor probabilidad de postular a áreas relacionadas con esta prueba, cuando se trata de las carreras más selectivas, el efecto positivo de los puntajes en la postulación es de mayor magnitud en los hombres. Una posible interpretación de este resultado, en línea con la literatura, es que las mujeres suelen tener menos confianza sobre sus propios conocimientos y aunque tengan buenos resultados, no eligen necesariamente las carreras más selectivas. 


\section{COMENTARIOS FINALES}

Los resultados de estas investigaciones son relevantes porque al tener las mujeres peores resultados en las pruebas de matemáticas, se alejan de las carreras en las que esta disciplina juega un rol fundamental. Estas diferencias en la participación de mujeres en carreras del área STEM tienen su correlato en brechas salariales por género, ya que significa que las mujeres tienden a estar subrepresentadas en las profesiones mejor pagadas en el mercado laboral, como Ingeniería y Ciencias Informáticas, concentrándose más bien en carreras del área de ciencias sociales y educación, que reciben menores salarios. Aumentar la participación de las mujeres en carreras STEM es importante para reducir brechas salariales y de género en general (OECD, 2015).

Todo esto evidencia la necesidad de tener políticas que promuevan la igualdad de género, teniendo en consideración que los sesgos de género son inconscientes. En este sentido, es importante hacer campañas y actividades de sensibilización, puesto que ayudan a tomar conciencia. De hecho, el movimiento feminista del año 2018 -protagonizado por las estudiantes universitarias- permitió que una gran mayoria de la población se interiorizara de estos sesgos.

Es fundamental también incluir el tema de género de forma transversal en las mallas curriculares de las carreras de Pedagogía, así como en la formación en servicio de los docentes. Junto con esto, también es importante revisar los libros de texto, porque en ellos también se observan estereotipos de género. Es relevante, además, generar conciencia en los padres y madres que deben apoyar tanto a sus hijos como a sus hijas en relación a sus aspiraciones profesionales. Hay que comenzar a producir cambios desde la primera infancia: la tarea es abrirles el mundo a niñas y niños, pues a ellos también se les debe ampliar su mirada y decirles que pueden ser un aporte en las carreras que hoy son consideradas femeninas.

A nivel de educación superior, es necesario promover el ingreso de mujeres a carreras STEM, así como incentivar de igual forma el ingreso de hombres a carreras hoy feminizadas. Un excelente ejemplo del tipo de política que se puede implementar es el Programa de Ingreso Prioritario de Equidad de Género (PEG) para el ingreso al Plan Común de la Escuela de Ingeniería y Ciencias de la Facultad de Ciencias Físicas y Matemáticas (FCFM) de la Universidad de Chile, iniciativa que existe desde el año 2013. Se trata de un programa que se ha propuesto potenciar la participación femenina en sus aulas, incentivando el ingreso de mujeres a la FCFM a través de 40 cupos especiales para mujeres que queden en lista de espera (es decir, bajo el último postulante seleccionado en el proceso regular del SUA). Este programa ha sido exitoso y ha aumentado el porcentaje de mujeres en primer año desde alrededor de un $19 \%$ a $32 \%$ en el año 2018, aumento que no se explica sólo por los 40 cupos 
adicionales sino porque más mujeres postularon a Ingeniería y quedaron aceptadas por sobre el puntaje de corte, todas con muy buenos resultados académicos.

También es relevante evaluar la incorporación de nuevos indicadores de desempeño en el proceso de selección universitaria, que tiendan a compensar los sesgos de género que tienen las pruebas estandarizadas competitivas y de alto impacto.

Asimismo, es importante facilitar y promover mayor participación de mujeres en el mundo científico, en política y en altos cargos en las empresas, ya que los roles ejercen un efecto relevante sobre niños y niñas.

El caso de la Facultad de Ciencias Físicas y Matemáticas de la Universidad de Chile muestra el efecto de las cuotas en lograr una distribución más equitativa del poder entre hombres y mujeres. Otros ejemplos con resultados positivos son el caso del Sistema de Empresas Públicas, que incorporó la medida de tener al menos un $40 \%$ de mujeres en los directorios de empresas del Estado. Con ello, el porcentaje de mujeres en directorios de las 22 empresas agrupadas en este sistema llegó a un $42 \%$ en 2017. La ley de cuotas, que obligó a las coaliciones políticas a no superar el 60\% de candidatos de un género, cuadriplicó el número de candidatas mujeres respecto del año 2013 y más mujeres llegaron a la Cámara de Diputados y al Senado. Las cuotas son importantes para romper la inercia, ya que contar con más mujeres en espacios que se han naturalizado como masculinos permite evolucionar, tener más de ellas cambia la dinámica y esto hace que se abran nuevos espacios.

Abrir espacios para las mujeres es fundamental, pero esto no sólo beneficia a las mujeres sino que al conjunto de la sociedad, evitando que se pierdan talentos para el desarrollo de las ciencias, las matemáticas, la ingeniería y la tecnología. Estas ocupaciones STEM se benefician de la diversidad que aportan las experiencias de las mujeres; la capacidad de plantearse preguntas y encontrar respuestas se ve fortalecida en ambientes más diversos (Franklin, 2013). 


\section{REFERENCIAS}

Ahmed, W., Minnaert, A., Kuyper, H., \& van der Werf, G. (2012). Reciprocal relationships between math self-concept and math anxiety. Learning and Individual Dierences, 22(3): 385-389.

Andreescu, T., Gallian, J. A., Kane, J. M., \& Mertz, J. E. (2007). Cross-Cultural Analysis of Students with Exceptional Talent in Mathematical Problem Solving. American Mathematical Monthly, 55(10): 1248-1260.

Arcidiacono, P. 2004. Ability sorting and the returns to college major. Journal of Econometrics 121, (1-2): 343-375.

Arias, O., Mizala, A, y Meneses, F. (2017). "Brecha de género en matemáticas: el sesgo de las pruebas competitivas", mimeo CEA, Ingeniería Industrial, U. de Chile, Santiago.

Auwarter, A. E. \& Aruguete, M. S. (2008a). Effects of Student Gender and Socioeconomic Status on Teacher Perceptions. In The Journal of Educational Research. Volume 101(4): 242-246

Auwarter, A. E. \& Aruguete, M. S. (2008b). Counselors perceptions of students who vary in gender and socioeconomic status. In Social Psychology and Education. Volume 11(4): 389-395

Baker, M. \& Milligan, K. (2013). Boy-Girl Differences in Parental Time Investments: Evidence from Three Countries. NBER Working Paper No. 18893.

Bartolj, T. \& Polanec, S. (2012). "College major choice and ability: Why is general ability not enough". Economics of Education Review 31(6): 996-1016.

Bassi, M., Blumberg, R.L., \& Mateo-Berganza, M. (2016). Under the cloak of invisibility: gender bias in teaching practices and learning outcomes. Working Paper IADB, Washington DC.

Bertrand, M. (2011). New Perspectives on Gender. In Handbook of Labor Economics, Vol. 4b: 1543-1590. Elsevier.

Bharadwaj, P., De Giorgi, G., Hansen, D. R., \& Neilson, C. (2015). The Gender Gap in Mathematics: Evidence from a Middle Income Country. SSRN Electronic Journal.

Booth, A. \& Nolen, P. (2011). "Choosing to compete: how different are girls and boys?” Journal of Economic Behavior \& Organization 81 (2): 542-555.

Booth, A. \& Nolen, P. (2012). "Gender differences in risk behavior: does nurture matter?” The Economic Journal 122 (558): F56-F78. 
Bordon, P., Canals, C. \& Mizala, A. (2017). Gender differences in college major choice. The case of Chile, mimeo CEA, Ingeniería Industrial, U. de Chile, Santiago.

Buchmann, C., DiPrete, T. A., \& McDaniel, A. (2008). Gender Inequalities in Education. Annual Review of Sociology, 34(1): 319-337.

Carlin, J. B. (2005). Regression models for twin studies: a critical review. International Journal of Epidemiology, 34(5): 1089-1099.

Croson, R. \& Gneezy, U. (2009). Gender differences in preferences. Journal of Economic Literature 47 (2): 448-474.

Cvencek, D., Meltzo, A. N., \& Greenwald, A. G. (2011). Math-Gender Stereotypes in Elementary School Children. Child Development, 82(3): 766-779.

Datta Gupta, N., Poulsen, A., \& Villeval, M.C. (2005). Male and Female Competitive Behavior - Experimental Evidence. SSRN Electronic Journal, 33(0): 35.

De Paola, M., Ponzo, M., \& Scoppa, V. (2015). Gender Deferences in Attitudes Towards Competition: Evidence from the Italian Scientific Qualification. IZA Discussion Paper No. 8859.

DiPrete, T. A. \& Jennings, J. L. (2009). Social/Behavioral Skills and the Gender Gap in Early Educational Achievement. CPRC Working Paper No. 09-08.

Dreber, A., von Essen, E., \& Ranehill, E. (2011). Outrunning the gender gap boys and girls compete equally. Experimental Economics, 14(4): 567-582.

Dreber, A., von Essen, E., \& Ranehill, E. (2014). Gender and competition in adolescence: task matters. Experimental Economics, 17(1): 154-172.

Else-Quest, N. M., Hyde, J. S., \& Linn, M. C. (2010). Cross-national patterns of gender differences in mathematics: A meta-analysis. Psychological Bulletin, 136(1): 103-127.

Espinoza, A.M. \& Taut, S. (2016). El rol del género en las interacciones pedagógicas de aulas de matemática chilenas. Psykhe 25(2):1-18.

Favara, M. (2012). The Cost of Acting "Girly”: Gender Stereotypes and Educational Choices. IZA DP N 7037, noviembre.

Filippin, A. \& Paccagnella, M. (2012). Family background, self-confidence and economic outcomes. Economics of Education Review, 31(5): 824-834.

Franklin, D. (2013). A Practical Guide to Gender Diversity for Computer Science Faculty. Synthesis Lectures on Professionalism and Career Advancement for Scientists and Engineers, 1(2):1-81. 
Fryer, R. G. \& Levitt, S. D. (2010). An Empirical Analysis of the Gender Gap in Mathematics. American Economic Journal: Applied Economics Vol. 2(2): 210-240.

Garratt, R. J., Weinberger, C. J., \& Johnson, N. (2013). The State Street Mile: Age and Gender Differences in Competition Aversion in the Field. Economic Inquiry, 51(1): 806-815.

Gneezy, U. \& Rustichini, A. (2004). Gender and competition at a young age. The American Economic Review 94 (2):377-381.

Gneezy, U., Leonard, K., \& List, J. A. (2009). Gender Differences in Competition: Evidence from a Matrilineal and a Patriarchal Society. Econometrica, 77(5): 1637-1664.

Gneezy, U., M. Niederle, \& Rustichini, A. (2003). "Performance in competitive environments: gender differences." Quarterly Journal of Economics 118 (3):1049-1074.

Gneezy, U. \& Rustichini, A. (2004). Gender and Competition at a Young Age. American Economic Review, 94(2): 377-381.

González de San Román, A. \& de la Rica Goiricelaya, S. (2012). Gender Gaps in PISA Test Scores: The Impact of Social Norms and the Mother's Transmission of Role Attitudes. IZA Discussion Papers No. 6338.

Guiso, L., F. Monte, P. Sapienza \& L. Zingales (2008) "Culture, gender and math" Science 320(5880): 1164-1165.

Gunderson, E. A., Gripshover, S. J., Romero, C., Dweck, C. S., Goldin-Meadow, S., \& Levine, S. C. (2013). Parent praise to 1-to 3-year-olds predicts children's motivational frameworks 5 years later. Child Development, 84(5): 1526-1541.

Gunderson, E. A., Ramirez, G., Levine, S. C., \& Beilock, S. L. (2012). The Role of Parents and Teachers in the Development of Gender-Related Math Attitudes. Sex Roles, 66(3-4): 153-166.

Hong, S., Yoo, S.-K., You, S., \& Wu, C.C. (2010). The Reciprocal Relationship between Parental Involvement and Mathematics Achievement: Autoregressive Cross-Lagged Modeling. Journal of Experimental Education, 78(4): 419-439.

Humlum, M.K., Kleinjans, K.J., and Nielsen, H.S. (2012). "An economic analysis of identity and career choice.” Economic Inquiry 50 (1): 39-61.

Hyde, J. S. (2014). Gender Similarities and Differences. Annual Review of Psychology, 65(1): 373-398. 
Hyde, J. S. \& Mertz, J. E. (2009). Gender, culture, and mathematics performance. Proceedings of the National Academy of Sciences, 106(22): 8801-8807.

Jurajda, Š. \& Münich, D. (2011). Gender Gap in Admission Performance under Competitive Pressure. American Economic Review Papers and Proceedings, 101(3): 514-518.

Kim, Y.-H., Chiu, C.-Y., \& Zou, Z. (2010). Know thyself: Misperceptions of actual performance undermine achievement motivation, future performance, and subjective well-being. Journal of Personality and Social Psychology, 99(3): 395-409.

Kurtz-Costes, B., Rowley, S.J., Harris-Britt, A. and Woods, T.A. (2008). Gender stereotypes about mathematics and science and self-perceptions of ability in late childhood and early adolescence. Merrill-Palmer Quarterly 54 (3): 386409 .

Lindberg, S. M., Hyde, J. S., Petersen, J. L., \& Linn, M. C. (2010). New trends in gender and mathematics performance: A meta-analysis. Psychological Bulletin, 136(6):1123-1135.

Maloney, E. A. \& Beilock, S. L. (2012). Math anxiety: Who has it, why it develops, and how to guard against it. Trends in Cognitive Sciences, 16(8): 404-406.

Mizala, A., Martinez, F., \& Martinez, S. (2015). Pre-service elementary school teachers' expectations about student performance: How their beliefs are affected by their mathematics anxiety and student's gender. Teaching and Teacher Education, 50: 70-78.

Nguyen, H.-H. D. \& Ryan, A. M. (2008). Does stereotype threat affect test performance of minorities and women? A meta-analysis of experimental evidence. Journal of Applied Psychology, 93(6): 1314-1334.

Niederle, M \& Vesterlund, L. (2007). Do women shy away from competition? Do men compete too much? Quarterly Journal of Economics 122 (3):1067-1101.

Niederle, M. \& Yestrumskas, A. H. (2008). Gender Differences in Seeking Challenges: The Role of Institutions. NBER WP \# 13922, Cambridge, MA.

Niederle, M. \& Vesterlund, L. (2010). Explaining the gender gap in math test scores: the role of competition. The Journal of Economic Perspectives 24 (2):129144.

Niederle, M. \& Vesterlund, L. (2011). Gender and Competition. Annual Review of Economics, 3(1): 601-630. 
Noe, C. (2010). Family background and female's choice of male's subjects: evidence from Italy. Child Working Paper $\mathrm{N}^{\circ} 1$.

OECD (2014). PISA 2012 Results: What Students Know and Can Do. Student Performance in Mathematics, Reading and Science (Volume I, Revised edition, February 2014). OECD Publishing.

OECD (2015). The ABC of Gender Equality in Education: Aptitude, Behavior, Confidence. PISA. OECD Publishing.

Örs, E., Palomino, F. \& Peyrache, E. (2013), Performance Gender-Gap: Does Competition Matter? Journal of Labor Economics 31(3): 443-499.

Paredes, V. (2014). A teacher like me or a student like me? Role model versus teacher bias effects. Economics of Education Review 39: 38-49.

Richardson, F. C. \& Suinn, R. M. (1972). The mathematics anxiety rating scale: psychometric data. Journal of Counseling Psychology, 19(6): 551-554.

Sikora, J. \& Pokropek, A. (2012a). Gender segregation of adolescent science career plans in 50 countries. Science Education DOI 10.1002/sce.20479 Published online 9 February 2012 in Wiley Online Library (wileyonlinelibrary.com).

Sikora, J. \& Pokropek, A. (2012b). Intergenerational transfers of preferences for science careers in comparative perspective. International Journal of Science Education 34(16): 2501-2527.

Steele, C. M. \& Aronson, J. (1995). Stereotype threat and the intellectual test performance of African Americans. Journal of Personality and Social Psychology, 69(5): 797-811.

Steinmayr, R., Crede, J., McElvany, N., \& Wirthwein, L. (2016). Subjective WellBeing, Test Anxiety, Academic Achievement: Testing for Reciprocal Effects. Frontiers in Psychology, 6.

Sutter, M. \& Rützler, D. (2010). Gender Differences in Competition Emerge Early in Life. IZA Discussion Paper No. 5015.

Sutter, M. \& Rützler, D. (2010). Gender differences in competition emerge early in life. IZA Discussion Paper N 5015.

Tomasetto, C., Mirisola, A., Galdi, S., \& Cadinu, M. (2015). Parents' math-gender stereotypes, children's self-perception of ability, and children's appraisal of parents' evaluations in 6-year-olds. Contemporary Educational Psychology, 42: 186-198.

Tournaki, N. (2003). Effect of Student Characteristics on Teachers' Predictions of Student Success. In The Journal of Educational Research. Volume 96(5): 310-319 
Turner, S.E. \& Bowen, W.G. (1999). Choice of major: the changing (unchanging) gender gap. Industrial and Labor Relations Review 52 (2): 289-313.

Walton, G. M. \& Spencer, S. J. (2009). Latent Ability: Grades and Test Scores Systematically Underestimate the Intellectual Ability of Negatively Stereotyped Students. Psychological Science, 20(9): 1132-1139.

Zahn-Waxler, C., Shirtcli, E. A., \& Marceau, K. (2008). Disorders of Childhood and Adolescence: Gender and Psychopathology. Annual Review of Clinical Psychology, 4(1): 275-303.

Zafar, B. (2009). College major choice and the gender gap, Staff Reports 364, Federal Reserve Bank of New York. 


\section{ANEXO}

Diseño de investigación casos hipotéticos de estudiantes

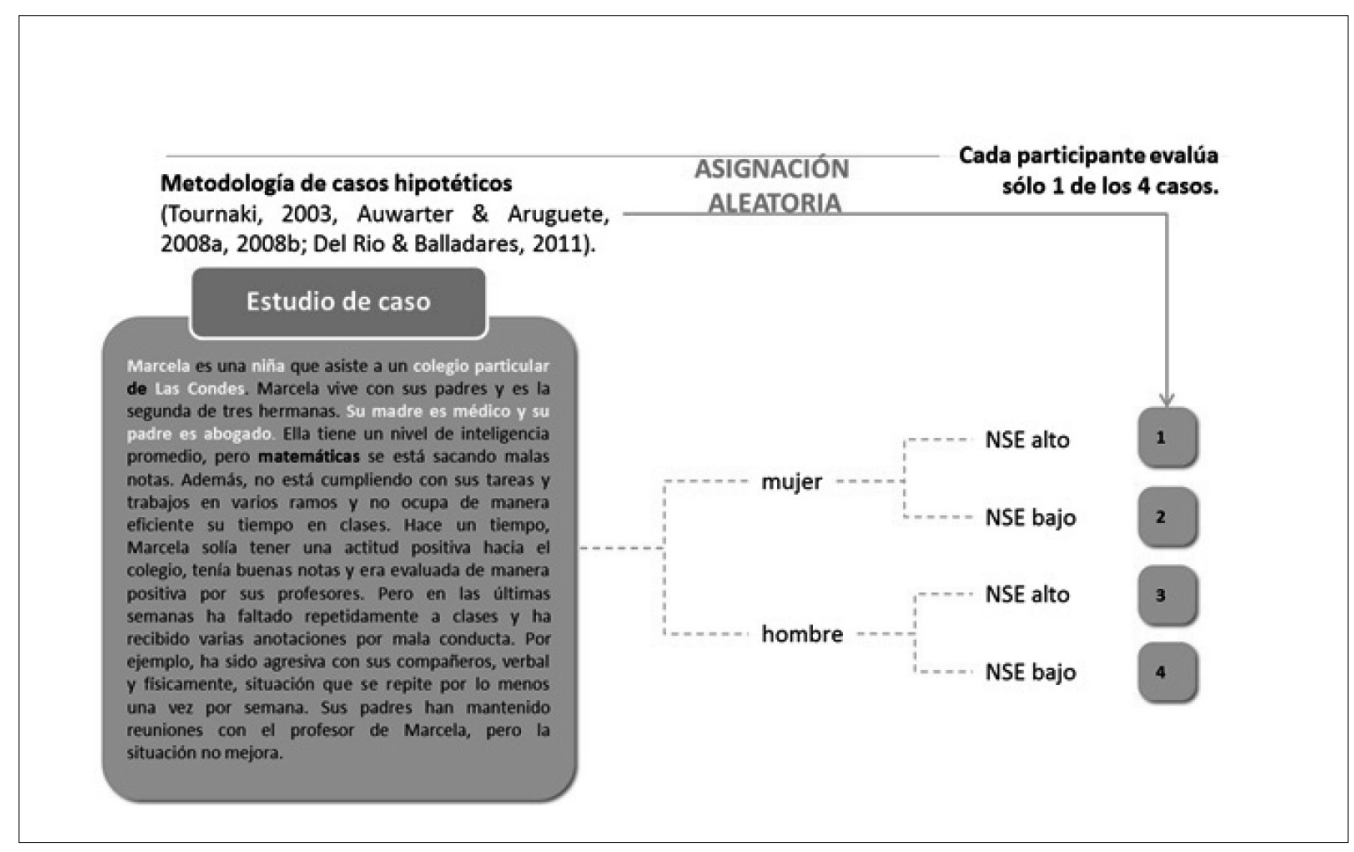

